\title{
Simulation Tools for Mobile Ad-hoc Sensor Networks: A State-of-the-Art Survey
}

\author{
Mohamad Nazim Jambli*, Halikul Lenando*, Kartinah Zen*, Sinarwati Mohamad Suhaili ${ }^{\dagger}$, Alan Tully \\ *Faculty of Computer Science \& Information Technology, Universiti Malaysia Sarawak, Malaysia \\ ${ }^{\dagger}$ Pre-University Studies, Universiti Malaysia Sarawak, Malaysia \\ ${ }^{\ddagger}$ School of Computing Science, Newcastle University, UK
}

\begin{abstract}
Mobile ad-hoc sensor networks (MASNETs) have recently become an important area of research for Mobile adhoc networks (MANETs) researchers. The increasing capabilities and the decreasing costs of sensors make MASNETs applications possible to be deployed in real world. However, before such application can be deployed, their performance need to be measured first. But, the use of real-world measurement is costly and time consuming. Therefore, it is more economical and practical to use simulation tools to simulate MASNETs applications. Although, there are many existing simulation tools for MANETs, most of them are not suitable for MASNETs due to resource-constraint of such networks. Therefore, it is essential to have a survey of the existing simulation tools for MASNETs. In this paper, the comparative study on different simulation tools is conducted to identify the most appropriate tool for MASNETs.
\end{abstract}

Index Terms-Simulation tools, Network simulator, Mobile wireless sensor networks

\section{INTRODUCTION}

Mobile wireless sensor networks (MWSNs) have recently become an important area of research for WSN researchers. The increasing capabilities and the decreasing costs of mobile sensors make MWSN applications such as Ocean Monitoring [1], Cattle [2], LISTSENse [3], CenWits [4] and PinPtr [5] become possible and practical to be implemented in real mobile environment. In this type of networks, mobility plays a key role in the deployment of these application. Furthermore, recent studies show that many researchers have proposed mobility-based routing protocols [6] for MWSNs to support mobile applications. Most of these protocols are compared and evaluated through simulation because it is very difficult to duplicate the real world such as in battle field. Thus, the use of real-world measurement is currently almost impossible, certainly costly and time consuming. Therefore, it is more economical and practical to use simulation tools to simulate MWSN applications in mobile environment and to create a statistically significant amount of test runs.

There are many network simulation tools currently available for studying WSNs including GloMoSim, OPNET, ns-2 [7], but choosing the right one for a given MWSN application is very important. To make this choice easier, we conduct a survey of several commonly used simulators that we find significant and interesting. In this paper, we present the results of the comparative study on different WSN simulation tools for evaluating routing protocols in MWSNs. In the second section, we present our methodology and evaluation criteria. In the third section, we describe and compare selected simulation tools according to the methodology and evaluation criteria. In the fourth section, we further discuss our findings, and in the fifth section, we conclude the paper and outlines the future work.

\section{Methodology And CRiteria}

There are many different possible platforms for simulation and evaluating of routing protocols for WSNs. Several studies have been done in comparing different simulators for WSNs as in [8], [9], [10], [7], [11], [12]. But some of the presented information is outdated and most of these studies are not focus on simulation tools for MWSNs where a mobility is one of the important factors that need to be considered. In contrast to that, we make a more detailed survey of WSNs simulators that most appropriate for MWNs. We define a set of criteria to evaluate and compare the selected simulation tools. These criteria can be distinguished into mandatory and optional criteria based on the requirement priority. The mandatory criteria are the main requirements of simulation tools, and it will be more convenient if the simulation tools can also satisfied the optional criteria. The following are the evaluation criteria that have been identified and classified into mandatory and optional criteria.

\section{A. Mandatory Criteria}

The mandatory criteria are the main requirements that are essential for any simulation tools to be able to simulate MWSNs. These criteria are evaluated on a yes $(\mathcal{V}) /$ no $(\boldsymbol{X})$ basis. Simulation tools that fail to meet all the required criteria are given no further consideration. The mandatory evaluation criteria are designed so that a yes $(\boldsymbol{V}) /$ no $(\boldsymbol{X})$ is easy to determine. There are three mandatory criteria identified for the best selection of MWSN simulation tools as follows:

- WSN-Specific. The selected simulation tools should be designed to simulate specific WSN applications and not just as a general purpose tools. If the tools is not designed as a WSN-Specific simulation tool, it might not consider some unique characteristics of WSN which are needed to accurately simulate the real WSNs environment.

- Energy model. The selected simulation tools need to provide some sort of energy models that able to examine the energy consumption of each static or mobile sensor 